\title{
SUFFICIENT AND NECESSARY CONDITIONS FOR CONFORMALITY. PART II. ANALYTIC VIEWPOINT
}

\author{
Melkana A. Brakalova \\ Fordham University, Department of Mathematics \\ Bronx, New York 10543, U.S.A.; brakalova@fordham.edu
}

This paper is dedicated in loving memory to V. V. Alexandrov and A. A. Gol'dberg.

\begin{abstract}
This is the second of two papers devoted to the topic of conformality at a point and related notions in the plane. We derive representation formulas and estimates for the modules of families of curves that are images of circles, radial segments and arcs of spirals under a $\mu$-homeomorphism. We use them to convert the extremal-length type sufficient and necessary conditions for conformality at a point from Part I to analytic sufficient conditions, that depend on directional dilatations and bypass the assumption of $K$-quaisconformality. Our results extend the Teichmüller-Wittich-Belinskii theorem, results of Reich and Walczak, the author and Jenkins, and Gutlyanskii and Martio.
\end{abstract}

\section{Definitions and main results}

Conformality at a point (see (1.3)) and related notions in the plane are rich properties that have had applications in the theory of Riemann surfaces, Nevanlinna theory, in the study of regularity properties of the boundary correspondence, local modulus of continuity properties and others (e.g. [10, 15, 16, 17, 22, 26]). Our main results, Theorems 1.2-1.4, provide sufficient conditions for conformality at a point and related notions for $A C L$-homeomorphisms, not necessarily $K$-quasiconformal, that depend on the directional dilatations in two or three directions.

Let $\Omega \subset \mathbf{C}$ be a domain and let $f$ be an $A C L$ (absolutely continuous on lines), sense-preserving homeomorphism $f: \Omega \rightarrow f(\Omega)$. Let $\mu$ be a Lebesgue-measurable complex-valued function in $\Omega \subset \mathbf{C}$ with $|\mu|<1$ a.e. and $\|\mu\|_{\infty} \leq 1 . \quad f$ is called a $\mu$-homeomorphism if

$$
f_{\bar{z}}=\mu f_{z}
$$

for a.e. $z \in \Omega$. Then $f$ has complex partial derivatives $f_{z}$ and $f_{\bar{z}}$ a.e., and the Jacobian $J_{f}=\left|f_{z}\right|^{2}-\left|f_{\bar{z}}\right|^{2} \geq 0$ a.e. In this paper we make the additional regularity assumptions that $f$ is locally absolutely continuous and $J_{f}>0$ a.e., thus $f$ is regular a.e. in $\Omega$.

Recall that $\mu$ is referred to as the Beltrami coefficient. If $\|\mu\|_{\infty}<1,(1.1)$ is referred to as the (complex) Beltrami equation. By the Existence theorem (measurable Riemann mapping theorem $[1,5,25])$ for such $\mu$ we can solve (1.1) and its solutions

doi:10.5186/aasfm.2010.3514

2000 Mathematics Subject Classification: Primary 30C62, 30C99.

Key words: Conformality at a point, Beltrami equation, quasiconformal mapping, degenerate Beltrami equation, $\mu$-homeomorphism, directional dilatation.

The work was partially supported by a Faculty Fellowship (Spring 2008) and a Faculty Research Grant, Fordham University. 
are quasiconformal mappings. If $\mu=0$ a.e. in $\Omega$ then $f$ is conformal in $\Omega$ [1, Corollary 1]. If $\|\mu\|_{\infty}=1$, one refers to (1.1) as the degenerate Beltrami equation. Under various conditions on $\mu$, one can solve the Beltrami equation in the degenerate case (see $[24,14,8,11,19,31]$ and others.) For the most recent research developments concerning solutions of the degenerate Beltrami equation in the plane, in higher dimensions and on metric spaces, see the recent monographs [20,27] and references therein.

One says that $z_{0} \in \Omega$ is a regular point if $f$ is totally differentiable at $z_{0}$, i.e. $f(z)=f\left(z_{0}\right)+f_{z}\left(z_{0}\right)\left(z-z_{0}\right)+f_{\bar{z}}\left(z_{0}\right)\left(\bar{z}-\bar{z}_{0}\right)+o\left(\left|z-z_{0}\right|\right)$ and $J_{f}\left(z_{0}\right)>0$. It is a well-known fact, in the theory of $K$-quasiconformal mappings, $[25,1]$, that an $A C L$ homeomorphism is totally differentiable a.e. At the regular points of $f$, the complex dilatation of $f$ is defined as $\mu_{f}=\frac{f_{\bar{z}}}{f_{z}}$, and the real dilatation as $D_{f}=\frac{1+\left|\mu_{f}\right|}{1-\left|\mu_{f}\right|}$, thus $D_{f} \geq 1$ at such a point. Without loss of generality we may assume that $D_{f}=1$ and $\mu_{f}=0$ outside of the set of regular points. Let $\alpha$ be a real number (the direction). At a regular point $z \in \Omega$ the directional dilatation $D_{f, \alpha}$ of $f$ in direction $\alpha$ is defined as

$$
D_{f, \alpha}=\frac{\left|f_{\alpha}\right|^{2}}{J_{f}}
$$

where $f_{\alpha}=f_{z}+e^{-2 i \alpha} f_{\bar{z}}$ is the directional derivative of $f$ in direction $\alpha$. Note that a.e. in $\Omega, \frac{1}{D_{f}} \leq D_{f, \alpha} \leq D_{f}$ and that $D_{f, \alpha}$ may assume any value in the interval $(0, \infty)$. Check $(3.1)$ for an equivalent expression to $(1.2)$.

Further we normalize $f$ so that it is defined in a neighborhood of the origin, say the unit disk, $U=\{z:|z|<1\}, f(0)=0$. We say that $f$ is conformal at the point 0 (at the origin) if

$$
\lim _{z \rightarrow 0} \frac{f(z)}{z}=A \neq 0, \infty
$$

Our main results on conformality hold under an additional assumption that:

Condition 1.1. Let $t>1$ be fixed. There exists a constant $C_{0}=C_{0}(t)$ such that

$$
\limsup _{r \rightarrow 0} \iint_{r<|z|<t r} \frac{1}{1-\left|\mu_{f}\right|^{2}} \frac{d A_{z}}{|z|^{2}}<C_{0}(t)<\infty .^{1}
$$

The main results of this paper are as follows.

Theorem 1.2. If

$$
\lim _{r \rightarrow 0} \iint_{r<|z|<1}\left(D_{f, \theta+\alpha}-1\right) \frac{d A_{z}}{|z|^{2}}
$$

\footnotetext{
${ }^{1}$ Instead of the integral condition involving $\mu_{f}$ one could consider a weaker one, namely $\iint_{r<|z|<t r}\left|D_{f, \theta+\frac{\pi}{2} j}-1\right| \frac{d A_{z}}{|z|^{2}}=o(1), r \rightarrow 0, j=0,1$. It is clear that if Condition 1.1 holds for some $t>1$, then it holds for any other $t>1$, and that it holds in case $f$ is $K$-quasiconformal.
} 
is finite for $\alpha=0, \pi / 2$, then

$$
\lim _{z \rightarrow 0} \frac{|f(z)|}{|z|}=A_{0} \neq 0, \infty
$$

$f$ is asymptotically a rotation on circles at $z=0$, namely (1.8) holds, and $f$ preserves asymptotically radial segments of any fixed aspect ratio $t>1$ at the origin, namely (1.9) holds. ${ }^{2}$

Theorem 1.3. If

$$
\lim _{r \rightarrow 0} \iint_{r<|z|<1}\left(D_{f, \theta+\alpha}-1\right) \frac{d A_{z}}{|z|^{2}}
$$

is finite for $\alpha=0, \pi / 2$ and $\alpha=\alpha_{0}, \alpha_{0} \neq k \frac{\pi}{2}, k$ any integer, then $f$ is conformal at $z=0$.

Theorem 1.4. If (1.4) is finite for $\alpha=0, \frac{\pi}{2}$, and if for some fixed $\theta_{0}, \lim _{r \rightarrow 0} \arg f\left(r e^{i \theta_{0}}\right)-$ $\theta_{0}=$ const, then $f$ is conformal at $z=0$.

The above theorems extend several earlier results on conformality and related notions, within the class of $K$-quasiconformal mappings and to the larger class of $\mu$-homeomorphisms. Section 5 provides a detailed account of the results we have in mind and some examples. Note that Theorem 1.4 gives particularly weak conformality conditions, provided that $f$ is known to preserve a direction in the limit. Later on, in Theorems 4.4-4.6 and Theorem 4.9 we state the above results in equivalent forms that depend explicitly on the complex dilatation.

In the special case when $f$ is a smooth radial stretching, i.e. $f\left(r e^{i \theta}\right)=\rho(r) e^{i \theta}$, $\rho(0)=0, \rho(r)$ increasing and continuously differentiable,

$$
\iint_{U}\left(D_{f}-1\right) \frac{d A_{z}}{|z|^{2}}<\infty
$$

is a sufficient and necessary condition for conformality at a point (see [25, p. 221]). In the case of $K$-quasiconformal mappings, by the well-known Teichmüller-WittichBelinskii theorem (Theorem 5.1), (1.6) is a sufficient condition. However it is not necessary. It is impossible to find, in the general case, sufficient and necessary conditions for conformality that depend on the real dilatation alone. To show this, we consider two $K$-quasiconformal mappings in $U, f$ and $g, f(0)=0, g(0)=0$, such that $D_{f}=D_{g}$, or equivalently $\left|\mu_{f}\right|=\left|\mu_{g}\right|$ in $U$, and such that $f$ is conformal at 0 and $g$ is not. In Example 1.1, [12] we constructed a radial stretching $f\left(r e^{i \theta}\right)=\rho(r) e^{i \theta}$ defined in $U, f(0)=0$ such that $f$ is conformal at the origin and $\mu_{f}\left(r e^{i \theta}\right)=-\frac{1}{3} e^{2 i \theta}$. Define $g\left(r e^{i \theta}\right)=r e^{i\left(\theta+\frac{\sqrt{2}}{2} \log r\right)}, 0<r<1$, and $g(0)=0$. Since $\mu_{g}\left(r e^{i \theta}\right)=\frac{\sqrt{2} i}{4+\sqrt{2} i} e^{2 i \theta}$, it follows that $\left|\mu_{f}\right|=\left|\mu_{g}\right|$. Clearly $g$ is not conformal at the origin. Note that, since $f$ from Example 1.1, [12] is conformal at the origin and (1.4) is not finite for any $\alpha$, the conditions in Theorem 1.3 and Theorem 1.4 are not necessary for conformality either.

\footnotetext{
${ }^{2}(1.8)$ and (1.9) are introduced further in this section.
} 
Both sufficient and necessary conditions for $\mu$-homeomorhisms, in terms of modules of families of curves, are obtained in [12]. Some of these results are stated in section 2 and are used to prove our main theorems.

Notions related to the study of conformality at a point are introduced below. We say that a $\mu$-homeomorphism $f$ is circle-like at the point 0 if it maps circles centered at the origin onto "almost" such circles, namely

$$
\lim _{r \rightarrow 0} \frac{\max _{|z|=r}|f(z)|}{\min _{|z|=r}|f(z)|}=1,
$$

in other words the circular dilatation (see [25]), $H_{f}(0)=1$. A $\mu$-homeomorphism conformal at 0 is circle-like at 0 . The converse is not true, as the example $f=z|z|$ shows.

A $\mu$-homeomorphism $f$ is asymptotically a rotation on circles at the origin if (1.5) holds and if for an appropriate choice of a branch of the argument

$$
\arg f\left(r e^{i \theta_{2}}\right)-\arg f\left(r e^{i \theta_{1}}\right)-\left(\theta_{2}-\theta_{1}\right) \rightarrow 0 \quad \text { as } r \rightarrow 0,
$$

uniformly in $\theta_{1}, \theta_{2}$. This notion was introduced in [9]. A $\mu$-homeomorphism, conformal at 0 , is asymptotically a rotation on circles at 0 . However, the converse does not hold as the example $f(z)=z e^{-i \log |z|}, 0<|z|<1, f(0)=0$, shows.

A $\mu$-homeomorphism is weakly conformal if it is circle-like, i.e. (1.7) holds, and if for an appropriate choice of a branch of the argument, $f$ satisfies (1.8). This notion was introduced for $K$-quasiconformal maps in [18]. Weak conformality is a weaker notion than asymptotical rotation on circles and stronger than circle-like behavior.

Let $0<r_{2}<r_{1}<1$, and $\theta$ be a real number. Denote by $\sigma_{f, \theta}\left(r_{2}, r_{1}\right)$ the image under $f$ of the radial segment $\sigma_{\theta}\left(r_{2}, r_{1}\right)=\left\{z: r_{2}<|z|<r_{1}, \arg z=\theta\right\}$. We define the angular oscillation of $\sigma_{f, \theta}\left(r_{2}, r_{1}\right)$ at the origin as

$$
\omega_{\sigma_{f, \theta}}\left(r_{2}, r_{1}\right)=\sup _{\sigma_{f, \theta}\left(r_{2}, r_{1}\right)} \arg z-\inf _{\sigma_{f, \theta}\left(r_{2}, r_{1}\right)} \arg z .
$$

In the above definition we choose a single-valued, continuous determination of the argument. $\omega_{\sigma_{f, \theta}}\left(r_{2}, r_{1}\right)$ measures the deviation of $\sigma_{f, \theta}\left(r_{2}, r_{1}\right)$ from a segment on a line through the origin. $\omega_{\sigma_{f, \theta}}\left(r_{2}, r_{1}\right)=0$ if and only if $\sigma_{f, \theta}\left(r_{2}, r_{1}\right)$ is such a segment.

Let $t>1$, and $r$ be such that $0<t r<1$. We say that a $\mu$-homeomorphism $f$ preserves asymptotically radial segments of fixed aspect ratio t at 0 , if (1.7) holds and uniformly in $\theta$,

$$
\omega_{\sigma_{f, \theta}}(r, t r) \rightarrow 0 \quad \text { as } r \rightarrow 0 .
$$

A $\mu$-homeomorphism conformal at 0 preserves asymptotically radial segments of fixed aspect ratio $t$ at 0 . The radial stretching $f=z(1-\log |z|), 0<|z|<1, f(0)=0$, does that without being conformal.

The rest of the paper is structured as follows. In Section 2 we state the sufficient and necessary conditions for conformality at a point from [12] that are applied in this paper. In Section 3 we use directional dilatations to provide representation formulas and estimates for modules of families of curves that are images under a $\mu$-homeomorphism of radial segments, circular arcs and arcs of spirals. In Section 4 we prove Theorems 1.2-1.4 and derive equivalent results, Theorems 4.4-4.6, and Theorem 4.9 that depend explicitly on the complex dilatation. In Section 5 we give 
an overview of previously known results on conformality and related notions at a point and discuss how we extend them.

\section{Geometric results on conformality}

In this section we state the sufficient and necessary conditions for conformality at a point from [12] that are applied in this paper. For a definition of the module of a family of curves and related terminology, see [2, 21], [12, Definition 1.3].

Let $0<r_{2}<r_{1}<1 . A_{f}=A_{f}\left(r_{2}, r_{1}\right)$ denotes the image under $f$ of $A\left(r_{2}, r_{1}\right)=$ $\left\{z: r_{2}<|z|<r_{1}\right\} . M\left(A\left(r_{2}, r_{1}\right)\right), M\left(A_{f}\left(r_{2}, r_{1}\right)\right)$ are their modules, where $M\left(A\left(r_{2}, r_{1}\right)\right)$ $=\frac{1}{2 \pi} \log \frac{r_{1}}{r_{2}}$.

Let $\theta_{1}<\theta_{2} \leq \theta_{1}+2 \pi . Q\left(r_{2}, r_{1}, \theta_{1}, \theta_{2}\right)=\left\{z=r e^{i \theta}: r_{2}<r<r_{1}, \theta_{1}<\theta<\theta_{2}\right\}$ denotes a quadrilateral with a-sides the circular arcs. Its image under $f$ is denoted by $Q_{f}\left(r_{2}, r_{1}, \theta_{1}, \theta_{2}\right)$. The a-sides of $Q_{f}\left(r_{2}, r_{1}, \theta_{1}, \theta_{2}\right)$ are the images of the a-sides of $Q\left(r_{2}, r_{1}, \theta_{1}, \theta_{2}\right)$. Let $M\left(Q\left(r_{2}, r_{1}, \theta_{1}, \theta_{2}\right)\right) M\left(Q_{f}\left(r_{2}, r_{1}, \theta_{1}, \theta_{2}\right)\right)$ be the modules of the family of curves connecting the a-sides of each quadrilateral, respectively. Here $M\left(Q\left(r_{2}, r_{1}, \theta_{1}, \theta_{2}\right)\right)=\frac{\theta_{2}-\theta_{1}}{\log \frac{r_{1}}{r_{2}}}$.

Let $\beta$ be a fixed real number. For $\theta \in\left[\theta_{1}, \theta_{1}+2 \pi\right)$ we consider the arcs of logarithmic spirals of inclination $\beta, s_{\theta}^{\beta}\left(r_{2}, r_{1}\right)=\left\{z: z=r e^{i(-\beta \log r+\theta)}, r_{2}<r<\right.$ $\left.r_{1}, \arg z=\theta\right\}$. Then the family $S^{\beta}\left(r_{2}, r_{1}\right)=\bigcup_{\theta \in\left[\theta_{1}, \theta_{1}+2 \pi\right)} s_{\theta}^{\beta}\left(r_{2}, r_{1}\right)$ sweeps out $A\left(r_{2}, r_{1}\right)$. Usually, $\theta_{1}=0 . S_{f}^{\beta}\left(r_{2}, r_{1}\right)$ denotes its image, $M\left(S^{\beta}\left(r_{2}, r_{1}\right)\right)$ and $M\left(S_{f}^{\beta}\left(r_{2}, r_{1}\right)\right)$ the modules of the corresponding families of curves. As shown in Corollary 3.2, $M\left(S^{\beta}\left(r_{2}, r_{1}\right)\right)=\frac{2 \pi}{\left(1+\beta^{2}\right) \log \frac{r_{1}}{r_{2}}}$.

Theorem 2.1. [12, Lemma 4.1] Let $t>1$ be a fixed number. Assume that

$$
M\left(A_{f}\left(r_{2}, r_{1}\right)\right)-M\left(A\left(r_{2}, r_{1}\right)\right)=o(1) \quad \text { as } r_{1} \rightarrow 0,
$$

and

$$
\lim _{r \rightarrow 0} M\left(Q_{f}\left(r, t r, \theta_{1}, \theta_{2}\right)\right)=\frac{\theta_{2}-\theta_{1}}{\log t}
$$

uniformly in $\theta_{1}$ and $\theta_{2}$. Then $\lim _{z \rightarrow 0} \frac{|f(z)|}{|z|}=A_{0} \neq 0, \infty, f$ is asymptotically a rotation on circles at $z=0$, namely (1.8) holds, and $f$ preserves asymptotically radial segments of fixed aspect ratio $t>1$ at the origin, namely (1.9) holds. Conditions (2.1) and (2.2) are necessary for conformality at the origin.

Combining the results of Lemma 3.1, Lemma 4.1, and Lemma 5.2 from [12] one obtains the following result.

Theorem 2.2. Assume that (2.1) and (2.2) from Theorem 2.1 hold, and that for some $\beta \neq 0$ and for any $\varepsilon>0$ there exists $R=R(\varepsilon)$ such that for $0<r_{2}<r_{1}<R$

$$
\frac{1}{M\left(S_{f}^{\beta}\left(r_{2}, r_{1}\right)\right)}-\frac{1}{M\left(S^{\beta}\left(r_{2}, r_{1}\right)\right)}<\varepsilon .
$$


Then $f$ is conformal at $z=0$. Conditions (2.1) and (2.2) are necessary for conformality at the origin.

Theorem 2.3. [12, Theorem 1.2] Assume that (2.1) and (2.2) from Theorem 2.1 hold. If for some fixed $\theta_{0}, \lim _{r \rightarrow 0} \arg f\left(r e^{i \theta_{0}}\right)-\theta_{0}=$ const, then $f$ is conformal at $z=0$. The above conditions are necessary for conformality at the origin.

\section{Representation formulas and estimates for modules of families of curves and ring domains}

In this section we use directional dilatations to provide representation formulas and estimates for modules of families of curves that are images under a $\mu$ homeomorphism of radial segments, circular arcs and arcs of spirals.

Let $\alpha$ be a real number. Let $z=r e^{i \theta} \in U$ be a regular point. Then the directional dilatation (1.2) in direction $\alpha$ can be written as

$$
D_{f, \alpha}=\frac{\left|1+e^{-2 i \alpha} \mu_{f}\right|^{2}}{1-\left|\mu_{f}\right|^{2}} .
$$

For $\alpha=\theta, \theta+\pi / 2$ we have

$$
D_{f, \theta}=\frac{\left|1+e^{-2 i \theta} \mu_{f}\right|^{2}}{1-\left|\mu_{f}\right|^{2}}, \quad D_{f, \theta+\pi / 2}=\frac{\left|1-e^{-2 i \theta} \mu_{f}\right|^{2}}{1-\left|\mu_{f}\right|^{2}} .
$$

Since $f_{r}=e^{i \theta}\left(f_{z}+e^{-2 i \theta} f_{\bar{z}}\right)$ and $f_{\theta}=i r e^{i \theta}\left(f_{z}-e^{-2 i \theta} f_{\bar{z}}\right)$ it follows that $\left|f_{r}\right|^{2}=$ $\left|f_{z}\right|^{2}\left|1+e^{-2 i \theta} \mu_{f}\right|^{2},\left|f_{\theta}\right|^{2}=\left|f_{z}\right|^{2} r^{2}\left|1-e^{-2 i \theta} \mu_{f}\right|^{2}$. Thus we obtain the following equivalent expressions at a regular point $z=r e^{i \theta}$ :

$$
D_{f, \theta}=\frac{\left|f_{r}\right|^{2}}{J_{f}}, \quad D_{f, \theta+\frac{\pi}{2}}=\frac{\left|f_{\theta}\right|^{2}}{r^{2} J_{f}} .
$$

In the literature, $D_{f, \theta}$ has been referred to as the radial dilatation, and $D_{f, \theta+\pi / 2}$ as the angular dilatation, and denoted by $D_{-\mu}$ and $D_{\mu}$, respectively (see e.g. [18] and [19]).

In addition, we observe that from (3.2) follows that at the regular point $z=r e^{i \theta}$

$$
D_{f, \theta}-1=2 \frac{\left|\mu_{f}\right|^{2}+\Re\left(e^{-2 i \theta} \mu_{f}\right)}{1-\left|\mu_{f}\right|^{2}}, \quad D_{f, \theta+\frac{\pi}{2}}-1=2 \frac{\left|\mu_{f}\right|^{2}-\Re\left(e^{-2 i \theta} \mu_{f}\right)}{1-\left|\mu_{f}\right|^{2}} .
$$

Let $0<r_{2}<r<r_{1}<1,0<r<1$, and $\theta_{1}<\theta_{2} \leq \theta_{1}+2 \pi$. Denote by $\mathscr{C}_{r}\left(\theta_{1}, \theta_{2}\right)=\left\{z:|z|=r, \theta_{1}<\arg z<\theta_{2}\right\}$ a circular arc of the circle $\mathscr{C}_{r}=$ $\{z:|z|=r\}$. Let $C\left(r_{2}, r_{1}, \theta_{1}, \theta_{2}\right)=\bigcup_{r \in\left(r_{2}, r_{1}\right)} \mathscr{C}_{r}\left(\theta_{1}, \theta_{2}\right)$ be the family of circular arcs.

Denote their images by $\mathscr{C}_{f, r}, \mathscr{C}_{f, r}\left(\theta_{1}, \theta_{2}\right), C_{f}\left(r_{2}, r_{1}, \theta_{1}, \theta_{2}\right) . \quad M\left(C\left(r_{2}, r_{1}, \theta_{1}, \theta_{2}\right)\right)$ and $M\left(C_{f}\left(r_{2}, r_{1}, \theta_{1}, \theta_{2}\right)\right)$ are the corresponding modules. Note that

$$
M\left(C\left(r_{2}, r_{1}, \theta_{1}, \theta_{2}\right)\right)=\frac{\log \frac{r_{1}}{r_{2}}}{\theta_{2}-\theta_{1}} .
$$

For $\theta \in\left[\theta_{1}, \theta_{2}\right)$, we denote by $\sigma_{\theta}\left(r_{2}, r_{1}\right)=\left\{z: r_{2}<|z|<r_{1}, \arg z=\theta\right\} a$ radial segment in $A\left(r_{2}, r_{1}\right)$, and its image by $\sigma_{f, \theta}\left(r_{2}, r_{1}\right)$ (see Section 1.) Denote $\Sigma\left(r_{2}, r_{1}, \theta_{1}, \theta_{2}\right)=\bigcup_{\theta \in\left(\theta_{1}, \theta_{2}\right)} \sigma_{\theta}\left(r_{2}, r_{1}\right)$ the family of radial segments and the family of 
their images by $\Sigma_{f}\left(r_{2}, r_{1}, \theta_{1}, \theta_{2}\right) . M\left(\Sigma\left(r_{2}, r_{1}, \theta_{1}, \theta_{2}\right)\right)$ and $M\left(\Sigma_{f}\left(r_{2}, r_{1}, \theta_{1}, \theta_{2}\right)\right)$ are the modules of the corresponding families of curves. Note that

$$
M\left(\Sigma\left(r_{2}, r_{1}, \theta_{1}, \theta_{2}\right)\right)=\frac{\theta_{2}-\theta_{1}}{\log \frac{r_{1}}{r_{2}}} .
$$

Lemma 3.1. Let $z=r e^{i \theta} \in Q\left(r_{2}, r_{1}, \theta_{1}, \theta_{2}\right)$. If $D_{f, \theta+\pi / 2} \in L^{1}\left(A\left(r_{2}, r_{1}\right)\right)$, then

$$
M\left(C_{f}\left(r_{2}, r_{1}, \theta_{1}, \theta_{2}\right)\right)=\int_{r_{2}}^{r_{1}} \frac{d r}{r \int_{\theta_{1}}^{\theta_{2}} D_{f, \theta+\frac{\pi}{2}} d \theta} .
$$

If $D_{f, \theta} \in L^{1}\left(A\left(r_{2}, r_{1}\right)\right)$, then

$$
M\left(\Sigma_{f}\left(r_{2}, r_{1}, \theta_{1}, \theta_{2}\right)\right)=\int_{\theta_{1}}^{\theta_{2}} \frac{d \theta}{\int_{r_{2}}^{r_{1}} D_{f, \theta} \frac{d r}{r}} .
$$

Proof. We only show the validity of (3.5). The proof of (3.6) is done in a similar manner. We use properties of the Lebesgue integral (see [28]) that hold true due to our regularity assumptions, including that $f$ is locally absolutely continuous and regular a.e. When necessary one should consider that the equalities below hold only a.e. Consider

$$
\rho=\frac{\left|f_{\theta}\right|}{J_{f} \int_{\theta_{1}}^{\theta_{2}} \frac{\left|f_{\theta}\right|^{2}}{J_{f}} d \theta}=\frac{D_{f, \theta+\frac{\pi}{2}}}{\left|f_{\theta}\right| \int_{\theta_{1}}^{\theta_{2}} D_{f, \theta+\frac{\pi}{2}} d \theta}
$$

defined a.e. in $Q\left(r_{2}, r_{1}, \theta_{1}, \theta_{2}\right)$, and $\rho_{0}=\rho \circ f^{-1}$ defined a.e. in $Q_{f}\left(r_{2}, r_{1}, \theta_{1}, \theta_{2}\right)$. The $\rho_{0}$-length of $\mathscr{C}_{f, r}\left(\theta_{1}, \theta_{2}\right)$ defined for almost every $r \in\left(r_{2}, r_{1}\right)$ is

$$
l_{\rho_{0}}\left(\mathscr{C}_{f, r}\left(\theta_{1}, \theta_{2}\right)\right)=\int_{\mathscr{C}_{f, r}\left(\theta_{1}, \theta_{2}\right)} \rho_{0}|d w|=\int_{\theta_{1}}^{\theta_{2}} \rho\left|f_{\theta}\right| d \theta=1 .
$$

Thus $\rho_{0}$ is an admissible function for the module problem defined by the family of curves $C_{f}\left(r_{2}, r_{1}, \theta_{1}, \theta_{2}\right)$, where $C_{f}\left(r_{2}, r_{1}, \theta_{1}, \theta_{2}\right) \subset Q_{f}\left(r_{2}, r_{1}, \theta_{1}, \theta_{2}\right)$. The $\rho_{0}$-area of $Q_{f}\left(r_{2}, r_{1}, \theta_{1}, \theta_{2}\right)$ is

$$
\begin{aligned}
A_{\rho_{0}}\left(Q_{f}\left(r_{2}, r_{1}, \theta_{1}, \theta_{2}\right)\right) & =\iint_{Q_{f}\left(r_{2}, r_{1}, \theta_{1}, \theta_{2}\right)} \rho_{0}^{2} d A_{w}=\iint_{Q\left(r_{2}, r_{1}, \theta_{1}, \theta_{2}\right)} \rho^{2} J_{f} d A_{z} \\
& =\int_{r_{2}}^{r_{1}} \int_{\theta_{1}}^{\theta_{2}} \rho^{2} J_{f} r d r d \theta=\int_{r_{2}}^{r_{1}} \frac{r d r}{\int_{\theta_{1}}^{\theta_{2}} \frac{f_{\left.f_{\theta}\right|^{2}}}{J_{f}} d \theta}=\int_{r_{2}}^{r_{1}} \frac{d r}{r \int_{\theta_{1}}^{\theta_{2}} D_{f, \theta+\frac{\pi}{2}} d \theta} .
\end{aligned}
$$

Let $\rho_{0}^{\star}$ be any other admissible function for the module problem defined by the family of curves $C_{f}\left(r_{2}, r_{1}, \theta_{1}, \theta_{2}\right)$. Then

$$
l_{\rho_{0}^{\star}}\left(\mathscr{C}_{f, r}\left(\theta_{1}, \theta_{2}\right)\right)=\int_{\mathscr{C}_{f, r}\left(\theta_{1}, \theta_{2}\right)} \rho_{0}^{\star}|d w| \geq 1 .
$$


Since $\int_{\mathscr{C}_{f, r}\left(\theta_{1}, \theta_{2}\right)}\left(\rho_{0}^{\star}-\rho_{0}\right)|d w| \geq 0$,

$$
\left(\int_{\theta_{1}}^{\theta_{2}} \frac{\left|f_{\theta}\right|^{2}}{J_{f}} d \theta\right)^{-1} \int_{\theta_{1}}^{\theta_{2}}\left(\rho^{\star}-\rho\right)\left|f_{\theta}\right| d \theta \geq 0
$$

where $\rho^{\star}=\rho_{0}^{\star} \circ f$. Therefore

$$
\iint_{Q\left(r_{2}, r_{1}, \theta_{1}, \theta_{2}\right)}\left(\rho^{\star} \rho-\rho^{2}\right) J_{f} d A_{z} \geq 0
$$

and thus

$$
\iint_{Q_{f}\left(r_{2}, r_{1}, \theta_{1}, \theta_{2}\right)}\left(\rho_{0}^{\star} \rho_{0}-\rho_{0}^{2}\right) d A_{w} \geq 0 .
$$

The Cauchy-Schwarz inequality implies

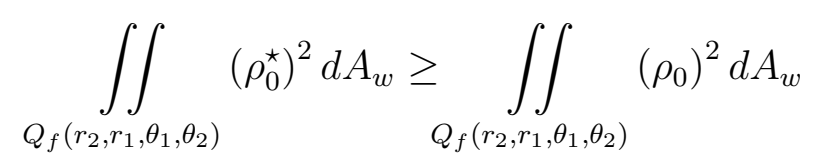

and therefore

$$
M\left(C_{f}\left(r_{2}, r_{1}, \theta_{1}, \theta_{2}\right)\right)=A_{\rho_{0}}\left(Q_{f}\left(r_{2}, r_{1}, \theta_{1}, \theta_{2}\right)\right)=\int_{r_{2}}^{r_{1}} \frac{d r}{r \int_{\theta_{1}}^{\theta_{2}} D_{f, \theta+\frac{\pi}{2}} d \theta} .
$$

Remark 3.2. From (3.3) follows that (3.5) and (3.6) can be rewritten in the following way. First,

$$
M\left(C_{f}\left(r_{2}, r_{1}, \theta_{1}, \theta_{2}\right)\right)=\int_{r_{2}}^{r_{1}} \frac{r d r}{\int_{\theta_{1}} \frac{\left|f_{\theta}\right|^{2}}{J_{f}} d \theta}
$$

and second,

$$
M\left(\Sigma_{f}\left(r_{2}, r_{1}, \theta_{1}, \theta_{2}\right)\right)=\int_{\theta_{1}}^{\theta_{2}} \frac{d \theta}{\int_{r_{2}}^{r_{1}} \frac{\left|f_{r}\right|^{2}}{J_{f}} \frac{d r}{r}} .
$$

The proof of Lemma 3.1 is similar to the one used by Rodin in [30] for the case of quadrilaterals, where $f$ is assumed to be sufficiently smooth. General results in this direction were obtained in the works of Andreian Cazacu (see [13] and the references to her earlier results therein.)

Corollary 3.1. Let $z=r e^{i \theta} \in Q\left(r_{2}, r_{1}, \theta_{1}, \theta_{2}\right), D_{f, \theta+\alpha} \in L^{1}\left(A\left(r_{2}, r_{1}\right)\right)$ for $\alpha=$ $0, \pi / 2$. Then

$$
\int_{\theta_{1}}^{\theta_{2}} \frac{d \theta}{\int_{r_{2}}^{r_{1}} D_{f, \theta} \frac{d r}{r}} \leq M\left(Q_{f}\left(r_{2}, r_{1}, \theta_{1}, \theta_{2}\right)\right) \leq \frac{1}{\left(\log \frac{r_{1}}{r_{2}}\right)^{2}} \iint_{Q\left(r_{2}, r_{1}, \theta_{1}, \theta_{2}\right)} D_{f, \theta+\frac{\pi}{2}} \frac{d A_{z}}{|z|^{2}}
$$




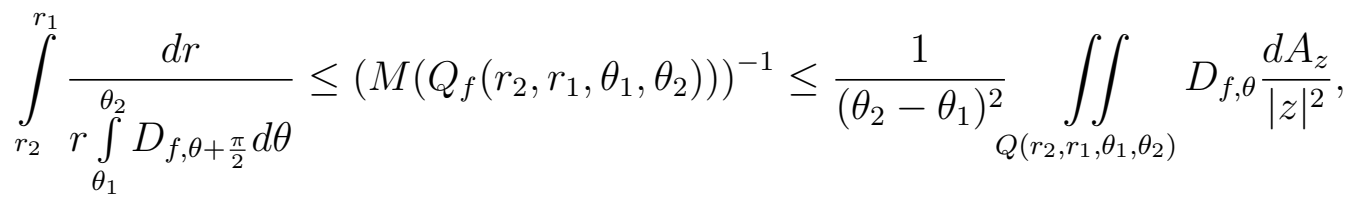

and

$$
\int_{r_{2}}^{r_{1}} \frac{d r}{r \int_{0}^{2 \pi} D_{f, \theta+\frac{\pi}{2}} d \theta} \leq M\left(A_{f}\left(r_{2}, r_{1}\right)\right) \leq \frac{1}{(2 \pi)^{2}} \iint_{A\left(r_{2}, r_{1}\right)} D_{f, \theta} \frac{d A_{z}}{|z|^{2}} .
$$

Proof. We have

$$
M\left(\Sigma_{f}\left(r_{2}, r_{1}, \theta_{1}, \theta_{2}\right)\right) \leq M\left(Q_{f}\left(r_{2}, r_{1}, \theta_{1}, \theta_{2}\right)\right) \leq\left(M\left(C_{f}\left(r_{2}, r_{1}, \theta_{1}, \theta_{2}\right)\right)\right)^{-1}
$$

and

$$
M\left(C_{f}\left(r_{2}, r_{1}, 0,2 \pi\right)\right) \leq M\left(A_{f}\left(r_{2}, r_{1}\right)\right) \leq\left(M\left(\Sigma_{f}\left(r_{2}, r_{1}, 0,2 \pi\right)\right)\right)^{-1}
$$

by the Comparison principle, see [1, 2]. Using (3.5), (3.6), and Cauchy-Schwarz inequality, Corollary 3.1 follows.

(3.9) is well-known if $f$ is a $K$-qusiconformal mapping (see [26, 29]), though the estimates are usually expressed in terms of the complex dilatation or partial derivatives. The upper estimate in (3.9) was proven for the general class of $\mu$-homeomorphisms by a different method in [19].

Let $\beta$ be a fixed real number. In the next two corollaries we give a representation formula and an estimate for the module of a family of arcs of logarithmic spirals of inclination $\beta$ and its image, defined in Section 2.

Corollary 3.2. We have

$$
M\left(S^{\beta}\left(r_{2}, r_{1}\right)\right)=\frac{2 \pi}{\left(1+\beta^{2}\right) \log \frac{r_{1}}{r_{2}}} .
$$

Proof. Let $z=r e^{i \theta}, 0 \leq \theta<2 \pi, h_{\beta}\left(r e^{i \theta}\right)=r e^{i(-\beta \log r+\theta)}$ be an area preserving map of $A\left(r_{2}, r_{1}\right)$ onto itself. By (3.3), $D_{h_{\beta, \theta}}=\frac{\left|\left(h_{\beta}\right)_{r}\right|^{2}}{J_{h_{\beta}}}=1+\beta^{2}$. Moreover,

$$
M\left(\Sigma_{h_{\beta}}\left(r_{2}, r_{1}, 0,2 \pi\right)\right)=\int_{0}^{2 \pi} \frac{d \theta}{\int_{r_{2}}^{r_{1}} D_{h_{\beta, \theta}} \frac{d r}{r}}
$$

by (3.6). Since $M\left(\Sigma_{h_{\beta}}\left(r_{2}, r_{1}, 0,2 \pi\right)\right)=M\left(S^{\beta}\left(r_{2}, r_{1}\right)\right)$, (3.10) follows.

Corollary 3.3. Assume that $D_{f, \theta+\alpha_{0}} \in L^{1}\left(A\left(r_{2}, r_{1}\right)\right)$, where $\alpha_{0}=-\tan ^{-1} \beta$. Then

$$
M\left(S_{f}^{\beta}\left(r_{2}, r_{1}\right)\right) \geq\left(\frac{\left(1+\beta^{2}\right)}{(2 \pi)^{2}} \iint_{A\left(r_{2}, r_{1}\right)} D_{f, \theta+\alpha_{0}} \frac{d A_{z}}{|z|^{2}}\right)^{-1} .
$$

Proof. Let $z=r e^{i \theta}, 0 \leq \theta<2 \pi$, and $h_{\beta}\left(r e^{i \theta}\right)=r e^{i(-\beta \log r+\theta)}$ be the map defined in the Corollary 3.2 above. Consider the map $g=f \circ h_{\beta}: A\left(r_{2}, r_{1}\right) \rightarrow A_{f}\left(r_{2}, r_{1}\right)$. 
Since $\left(h_{\beta}\right)_{z}+e^{-2 i \theta}\left(h_{\beta}\right)_{\bar{z}}=e^{-i \theta}\left(h_{\beta}\right)_{r}$ and $\left(\overline{h_{\beta}}\right)_{z}+e^{-2 i \theta}\left(\overline{h_{\beta}}\right)_{\bar{z}}=e^{-i \theta}\left(\overline{h_{\beta}}\right)_{r}$, one has

$$
\iint_{A\left(r_{2}, r_{1}\right)} D_{g, \theta} \frac{d A_{z}}{|z|^{2}}=\iint_{A\left(r_{2}, r_{1}\right)} \frac{\left|g_{z}+e^{-2 i \theta} g_{\bar{z}}\right|^{2}}{J_{g}} \frac{d A_{z}}{|z|^{2}}=\iint_{A\left(r_{2}, r_{1}\right)} \frac{\left|f_{\zeta}\left(h_{\beta}\right)_{r}+f_{\bar{\zeta}}\left(\overline{h_{\beta}}\right)_{r}\right|^{2}}{J_{g}} \frac{d A_{z}}{|z|^{2}}
$$

After change of variables $\zeta=h_{\beta}(z)$, using the identity $\frac{1+i \beta}{1-i \beta}=e^{-2 i \alpha_{0}}$, the fact that $J_{h_{\beta}}=1$, and $r\left(h_{\beta}\right)_{r}=\left(h_{\beta}\right)(1-i \beta), r\left(\bar{h}_{\beta}\right)_{r}=\left(\bar{h}_{\beta}\right)(1+i \beta)$, the last term in (3.12) is equal to

$$
\iint_{A\left(r_{2}, r_{1}\right)} \frac{\left(1+\beta^{2}\right)\left|f_{\zeta}+\frac{1+i \beta}{1-i \beta} f_{\bar{\zeta}} \frac{\bar{\zeta}}{\zeta}\right|^{2}}{J_{f}} \frac{d A_{\zeta}}{|\zeta|^{2}}=\iint_{A\left(r_{2}, r_{1}\right)} D_{f, \theta+\alpha_{0}} \frac{d A_{z}}{|z|^{2}} .
$$

Thus

$$
\iint_{A\left(r_{2}, r_{1}\right)} D_{g, \theta} \frac{d A_{z}}{|z|^{2}}=\iint_{A\left(r_{2}, r_{1}\right)} D_{f, \theta+\alpha_{0}} \frac{d A_{z}}{|z|^{2}}
$$

and $D_{g, \theta} \in L^{1}\left(A\left(r_{2}, r_{1}\right)\right)$. By (3.6) of Lemma 3.1 and the Cauchy-Schwarz inequality

$$
M\left(\Sigma_{g}\left(r_{2}, r_{1}, 0,2 \pi\right)\right) \geq\left(\frac{1}{(2 \pi)^{2}} \iint_{A\left(r_{2}, r_{1}\right)} D_{g, \theta} \frac{d A_{z}}{|z|^{2}}\right)^{-1} .
$$

Since $M\left(\Sigma_{g}\left(r_{2}, r_{1}, 0,2 \pi\right)\right)=M\left(S_{f}^{\beta}\left(r_{2}, r_{1}\right)\right)$, (3.11) follows from (3.13).

Corollary 3.4. Let $z=r e^{i \theta}$, and let $\beta$ be a fixed number. Assume that $D_{f, \theta+\alpha} \in$ $L^{1}\left(A\left(r_{2}, r_{1}\right)\right)$ for $\alpha=\frac{\pi}{2}, \alpha_{0}$, where $\alpha_{0}=-\tan ^{-1} \beta$. Then

$$
\int_{r_{2}}^{r_{1}} \frac{d r}{r \int_{0}^{2 \pi} D_{f, \theta+\frac{\pi}{2}} d \theta} \leq M\left(A_{f}\left(r_{2}, r_{1}\right)\right) \leq \frac{\left(1+\beta^{2}\right)}{(2 \pi)^{2}} \iint_{A\left(r_{2}, r_{1}\right)} D_{f, \theta+\alpha_{0}} \frac{d A_{z}}{|z|^{2}} .
$$

Proof. By the Comparison principle, $M\left(C_{f}\left(r_{2}, r_{1}, 0,2 \pi\right)\right) \leq M\left(A_{f}\left(r_{2}, r_{1}\right)\right) \leq$ $\left(M\left(S_{f}^{\beta}\left(r_{2}, r_{1}\right)\right)\right)^{-1}$. Using (3.5) and (3.11) we obtain (3.14).

(3.9) is a special case of (3.14) and the latter may provide, depending on the choice of $\beta$, more precise upper estimate for $M\left(A_{f}\left(r_{2}, r_{1}\right)\right)$.

\section{Proof of the main results}

In this section we prove Theorems $1.2-1.4$ and derive equivalent results, Theorems 4.4-4.6.

Lemma 4.1. Let $f$ be a $\mu$-homeomorphism, $D_{f, \theta+\alpha} \in L^{1}\left(A\left(r_{2}, r_{1}\right)\right)$ for $\alpha=$ $0, \pi / 2$. Denote by $Q=Q\left(r_{2}, r_{1}, \theta_{1}, \theta_{2}\right), Q_{f}=Q_{f}\left(r_{2}, r_{1}, \theta_{1}, \theta_{2}\right)$, and by $M\left(Q_{f}\right)$ the 
module of the latter. Then

$$
\left|\frac{1}{M\left(Q_{f}\right)}-\frac{\log \frac{r_{1}}{r_{2}}}{\theta_{2}-\theta_{1}}\right| \leq \frac{1}{\left(\theta_{2}-\theta_{1}\right)^{2}} \max _{j=0,1}\left|\iint_{Q}\left(D_{f, \theta+\frac{\pi}{2} j}-1\right) \frac{d A_{z}}{|z|^{2}}\right|
$$

and

$$
\left|M\left(Q_{f}\right)-\frac{\theta_{2}-\theta_{1}}{\log \frac{r_{1}}{r_{2}}}\right| \leq \frac{1}{\left(\log \frac{r_{1}}{r_{2}}\right)^{2}} \max _{j=0,1}\left|\iint_{Q}\left(D_{f, \theta+\frac{\pi}{2} j}-1\right) \frac{d A_{z}}{|z|^{2}}\right| .
$$

Proof. Let $m=\frac{\theta_{2}-\theta_{1}}{\log \frac{r_{1}}{r_{2}}}$. Then

$$
M\left(Q_{f}\right)-m \leq \frac{1}{\left(\log \frac{r_{1}}{r_{2}}\right)^{2}} \iint_{Q}\left(D_{f, \theta+\frac{\pi}{2}}-1\right) \frac{d A_{z}}{|z|^{2}}
$$

and

$$
\frac{1}{M\left(Q_{f}\right)}-\frac{1}{m} \leq \frac{1}{\left(\theta_{2}-\theta_{1}\right)^{2}} \iint_{Q}\left(D_{f, \theta}-1\right) \frac{d A_{z}}{|z|^{2}}
$$

follow from (3.7) and (3.8) of Corollary 3.1. This yields

$$
m-M\left(Q_{f}\right) \leq \frac{M\left(Q_{f}\right)}{m} \frac{1}{\left(\log \frac{r_{1}}{r_{2}}\right)^{2}} \iint_{Q}\left(D_{f, \theta}-1\right) \frac{d A_{z}}{|z|^{2}}
$$

and

$$
\frac{1}{m}-\frac{1}{M\left(Q_{f}\right)} \leq \frac{m}{M\left(Q_{f}\right)} \frac{1}{\left(\theta_{2}-\theta_{1}\right)^{2}} \iint_{Q}\left(D_{f, \theta+\frac{\pi}{2}}-1\right) \frac{d A_{z}}{|z|^{2}}
$$

Considering separately the case when $M\left(Q_{f}\right)-m>0$ (and therefore $\frac{m}{M\left(Q_{f}\right)}<1$ ) and the case when $M\left(Q_{f}\right)-m<0$ (and therefore $\frac{M\left(Q_{f}\right)}{m}<1$ ) from the above inequalities we obtain the desired estimates.

Lemma 4.2. Let $D_{f, \theta+\alpha} \in L^{1}\left(A\left(r_{2}, r_{1}\right)\right)$ for $\alpha=0, \pi / 2$. Then

$$
\left|M\left(A_{f}\left(r_{2}, r_{1}\right)\right)-\frac{\log \frac{r_{1}}{r_{2}}}{2 \pi}\right| \leq \frac{1}{(2 \pi)^{2}} \max _{j=0,1}\left|\iint_{A\left(r_{2}, r_{1}\right)}\left(D_{f, \theta+\frac{\pi}{2} j}-1\right) \frac{d A_{z}}{|z|^{2}}\right| .
$$

The proof of Lemma 4.2 is identical to the proof of Lemma 4.1.

Lemma 4.3. Let $f$ satisfy Condition 1.1. Let $t>1$ be a fixed number, $0<r<$ $\operatorname{tr}<1$, and $A(r, t r)=\{z: r<|z|<t r\}$. Assume that

$$
\iint_{A(r, t r)} \frac{\left|\mu_{f}\right|^{2}}{1-\left|\mu_{f}\right|^{2}} \frac{d A_{z}}{|z|^{2}}=o(1) \text { as } r \rightarrow 0 .
$$


Then

$$
\iint_{A(r, t r)} \frac{\left|\Re\left(e^{-2 i \theta} \mu_{f}\right)\right|}{1-\left|\mu_{f}\right|^{2}} \frac{d A_{z}}{|z|^{2}}=o(1) \text { as } r \rightarrow 0 .
$$

Proof. Using Cauchy-Schwarz inequality and Condition 1.1 we have

$$
\begin{aligned}
\left(\iint_{A(r, t r)} \frac{\left|\Re\left(e^{-2 i \theta} \mu_{f}\right)\right|}{1-\left|\mu_{f}\right|^{2}} \frac{d A_{z}}{|z|^{2}}\right)^{2} & \leq \iint_{A(r, t r)} \frac{\left|\mu_{f}\right|^{2}}{1-\left|\mu_{f}\right|^{2}} \frac{d A_{z}}{|z|^{2}} \iint_{A(r, t r)} \frac{1}{1-\left|\mu_{f}\right|^{2}} \frac{d A_{z}}{|z|^{2}} \\
& \leq C_{0}(t) \iint_{A(r, t r)} \frac{\left|\mu_{f}\right|^{2}}{1-\left|\mu_{f}\right|^{2}} \frac{d A_{z}}{|z|^{2}}
\end{aligned}
$$

for $r$ small enough, and (4.2) follows from (4.1).

Proof of Theorem 1.2. Let $0<r_{2}<r_{1}<1$. The assumptions in Theorem 1.2 imply

$$
\iint_{A\left(r_{2}, r_{1}\right)}\left(D_{f, \theta+\alpha}-1\right) \frac{d A_{z}}{|z|^{2}}=o(1) \text { as } r_{1} \rightarrow 0,
$$

for $\alpha=0, \pi / 2$. By Lemma 4.2

$$
\left|M\left(A_{f}\left(r_{2}, r_{1}\right)\right)-\frac{1}{2 \pi} \log \frac{r_{1}}{r_{2}}\right| \leq \frac{1}{(2 \pi)^{2}} \max _{j=0,1}\left|\iint_{A\left(r_{2}, r_{1}\right)}\left(D_{f, \theta+\frac{\pi}{2} j}-1\right) \frac{d A_{z}}{|z|^{2}}\right|
$$

and, due to (4.3), (2.1) holds:

$$
M\left(A_{f}\left(r_{2}, r_{1}\right)\right)-\frac{1}{2 \pi} \log \frac{r_{1}}{r_{2}}=o(1) \text { as } r_{1} \rightarrow 0 .
$$

Let $t>1$ be a fixed number. Denote for $0<r<t r<1, A(r, t r)=\{z: r<|z|<t r\}$. Next we show that for $j=0,1$,

$$
\iint_{A(r, t r)}\left|D_{f, \theta+\frac{\pi}{2} j}-1\right| \frac{d A_{z}}{|z|^{2}}=o(1) \text { as } r \rightarrow 0 .
$$

From (3.4) follows

$$
\iint_{Q\left(r, t r, \theta_{1}, \theta_{2}\right)}\left|D_{f, \theta+\frac{\pi}{2} j}-1\right| \frac{d A_{z}}{|z|^{2}} \leq 2 \iint_{A(r, t r)} \frac{\left|\mu_{f}\right|^{2}+\left|\Re\left(e^{-2 i \theta} \mu_{f}\right)\right|}{1-\left|\mu_{f}\right|^{2}} \frac{d A_{z}}{|z|^{2}} .
$$

Since (1.4) is finite for $\alpha=0, \pi / 2$, we have (4.1). Lemma 4.3 implies (4.2). From this and (4.5) follows (4.4). By Lemma 4.1

$$
\left|M\left(Q_{f}\left(r, \operatorname{tr}, \theta_{1}, \theta_{2}\right)\right)-\frac{\theta_{2}-\theta_{1}}{\log t}\right| \leq \frac{1}{(\log t)^{2}} \max _{j=0,1}\left(\iint_{A(r, t r)}\left|D_{f, \theta+\frac{\pi}{2} j}-1\right| \frac{d A_{z}}{|z|^{2}}\right) .
$$

Using (4.4) we have (2.2)

$$
M\left(Q_{f}\left(r, t r, \theta_{1}, \theta_{2}\right)\right)-\frac{\theta_{2}-\theta_{1}}{\log t}=o(1) \text { as } r \rightarrow 0
$$


uniformly in $\theta_{1}, \theta_{2}$. Thus the assumptions of Theorem 1.2 imply (2.1) and (2.2) of Theorem 2.1. Theorem 1.2 follows.

Proof of Theorem 1.3. From the assumptions in Theorem 1.3 and the proof of Theorem 1.2 follow (2.1) and (2.2) of Theorem 2.2. We only need to show (2.3). Take $\beta=-\tan \alpha_{0}$. Since (1.4) is finite for $\alpha=\alpha_{0}$, for any sufficiently small $\varepsilon>0$ we can choose $R=R(\varepsilon)$ such that for $0<r_{2}<r_{1}<R$,

$$
\frac{1+\beta^{2}}{(2 \pi)^{2}}\left|\iint_{A\left(r_{2}, r_{1}\right)}\left(D_{f, \theta+\alpha_{0}}-1\right) \frac{d A_{z}}{|z|^{2}}\right|<\varepsilon .
$$

From (3.11) of Corollary 3.3 follows that

$$
\frac{1}{M\left(S_{f}^{\beta}\left(r_{2}, r_{1}\right)\right)}-\frac{\left(1+\beta^{2}\right) \log \frac{r_{1}}{r_{2}}}{2 \pi} \leq \frac{1+\beta^{2}}{(2 \pi)^{2}} \iint_{A\left(r_{2}, r_{1}\right)}\left(D_{f, \theta+\alpha_{0}}-1\right) \frac{d A_{z}}{|z|^{2}} .
$$

Therefore, by Corollary 3.2, (2.3) holds. Theorem 1.3 follows from Theorem 2.2 and thus $f$ is conformal at $z=0$.

Proof of Theorem 1.4. From the assumptions in Theorem 1.4 and by Theorem 1.2, $\lim _{z \rightarrow 0} \frac{|f(z)|}{|z|}=A_{0} \neq 0, \infty$ and $\arg f\left(r e^{i \theta_{2}}\right)-\arg f\left(r e^{i \theta_{1}}\right)-\left(\theta_{2}-\theta_{1}\right) \rightarrow 0$ as $r \rightarrow 0$. Since for some fixed $\theta_{0}, \lim _{r \rightarrow 0} \arg f\left(r e^{i \theta_{0}}\right)-\theta_{0}=$ const, $f$ is conformal at the origin.

The following Theorems 4.4-4.6 are equivalent to Theorems 1.2-1.4, respectively.

\section{Theorem 4.4. If}

$$
\begin{gathered}
\iint_{U} \frac{\left|\mu_{f}\right|^{2}}{1-\left|\mu_{f}\right|^{2}} \frac{d A_{z}}{|z|^{2}}<\infty \\
\iint_{U} \frac{\Re\left(e^{-2 i \theta} \mu_{f}\right)}{1-\left|\mu_{f}\right|^{2}} \frac{d A_{z}}{|z|^{2}}
\end{gathered}
$$

exists in the sense of principal value, then $\lim _{z \rightarrow 0} \frac{|f(z)|}{|z|}=A_{0} \neq 0, \infty, f$ is asymptotically a rotation on circles at $z=0$, namely (1.8) holds, and $f$ preserves asymptotically radial segments of any fixed aspect ratio $t>1$ at the origin, namely (1.9) holds.

Theorem 4.5. If (4.6) holds and

$$
\iint_{U} \frac{\mu_{f}}{1-\left|\mu_{f}\right|^{2}} \frac{d A_{z}}{z^{2}}
$$

exists in the sense of principal value, then $f$ is conformal at $z=0$.

Theorem 4.6. If (4.6) holds, (4.7) exists in the sense of principal value, and if for some fixed $\theta_{0}, \lim _{r \rightarrow 0} \arg f\left(r e^{i \theta_{0}}\right)-\theta_{0}=$ const, then $f$ is conformal at $z=0$.

The next results establish the equivalence between Theorems 1.2-1.4 and Theorems 4.4-4.6, respectively. 
Lemma 4.7. If

$$
\lim _{r \rightarrow 0} \iint_{r<|z|<1} \frac{\Re\left(e^{-2 i(\theta+\alpha)} \mu_{f}\right)}{1-\left|\mu_{f}\right|^{2}} \frac{d A_{z}}{|z|^{2}}
$$

is finite ${ }^{3}$ for any two real numbers $\alpha=\alpha_{1}, \alpha=\alpha_{2}, \alpha_{1}-\alpha_{2} \neq k \frac{\pi}{2}, k$ any integer, then (4.9) is finite for any $\alpha$.

Proof. The proof follows from the properties of the Lebesgue integral and the elementary identity $\Re\left(z e^{-2 i \alpha}\right)=a \Re\left(z e^{-2 i \alpha_{1}}\right)+b \Re\left(z e^{-2 i \alpha_{2}}\right)$, valid for any $z$. Here $a=\frac{\sin \left(2\left(\alpha-\alpha_{2}\right)\right)}{\sin \left(2\left(\alpha_{1}-\alpha_{2}\right)\right)}, b=\frac{\sin \left(2\left(\alpha_{1}-\alpha\right)\right)}{\sin \left(2\left(\alpha_{1}-\alpha_{2}\right)\right)}$.

Theorem 4.8. Let $\arg z=\theta$. First, the limit

$$
\lim _{r \rightarrow 0} \iint_{r<|z|<1}\left(D_{f, \theta+\alpha}-1\right) \frac{d A_{z}}{|z|^{2}}
$$

is finite for $\alpha=0, \pi / 2$ if and only if (4.6) holds and

$$
\iint_{U} \frac{\Re\left(e^{-2 i \theta} \mu_{f}\right)}{1-\left|\mu_{f}\right|^{2}} \frac{d A_{z}}{|z|^{2}}
$$

exists in the sense of principal value.

Second, the limit (4.10) is finite for $\alpha=0, \pi / 2$ and $\alpha_{0}, \alpha_{0} \neq k \frac{\pi}{2}, k$ an integer, if and only if (4.6) holds and

$$
\iint_{U} \frac{\mu_{f}}{1-\left|\mu_{f}\right|^{2}} \frac{d A_{z}}{z^{2}}
$$

exists in the sense of principal value.

Proof. The first sufficient and necessary part of Theorem 4.8 follows immediately from (3.4).

Now we prove the necessity in the second part of the theorem. Since (4.10) is finite for $\alpha=0, \pi / 2$ and $\alpha_{0}, \alpha_{0} \neq k \frac{\pi}{2}, k$ an integer, then (4.6) holds and

$$
\iint_{U} \frac{\Re\left(e^{-2 i(\theta+\alpha)} \mu_{f}\right)}{1-\left|\mu_{f}\right|^{2}} \frac{d A_{z}}{|z|^{2}}
$$

exists in the sense of principal value for $\alpha=0, \alpha_{0}, \alpha_{0} \neq k \frac{\pi}{2}, k$ any integer. By Lemma 4.7,

$$
\iint_{U} \frac{\Re\left(e^{-2 i(\theta+\alpha)} \mu_{f}\right)}{1-\left|\mu_{f}\right|^{2}} \frac{d A_{z}}{|z|^{2}}
$$

exists in the sense of principal value for any $\alpha$, in particular $\alpha=\frac{\pi}{4}$, and this implies that (4.12) exists in the sense of principal value. The necessity follows. The sufficient part can be proven in a similar manner.

The above considerations yield that the following sufficient condition for conformality is equivalent to Theorem 4.5.

3i.e. $\iint_{U} \frac{\Re\left(e^{-2 i(\theta+\alpha)} \mu_{f}\right)}{1-\left|\mu_{f}\right|^{2}} \frac{d A_{z}}{|z|^{2}}$ exists in the sense of principal value. 
Theorem 4.9. If (4.6) holds and (4.13) exists in the sense of principal value for $\alpha=\alpha_{1}, \alpha_{2}, \alpha_{1}-\alpha_{2} \neq k \frac{\pi}{2}, k$ an integer, then $f$ is conformal at $z=0$.

\section{Some comments on the history of the study of conformality at a point}

In this section we give an account of previously known results on conformality at a point and how we extend these results. First we state the Teichmüller-WittichBelinskii theorem.

Theorem 5.1. [3, 32, 33] Let $f$ be a quasiconformal mapping in $U$ such that $f(0)=0$. If

$$
\iint_{U}\left(D_{f}-1\right) \frac{d A_{z}}{|z|^{2}}<\infty
$$

then $f$ is conformal at 0 .

Remark 5.2. (5.1) can be rewritten in the equivalent form

$$
\iint_{U}\left|\mu_{f}\right| \frac{d A_{z}}{|z|^{2}}<\infty
$$

The proof of Theorem 5.1 was accomplished in several stages. First, in 1938, Teichmüller [32] showed that

$$
\lim _{z \rightarrow 0} \frac{|f(z)|}{|z|}=A_{0} \neq 0, \infty
$$

under the assumption that $f$ is a diffeomorphism and satisfies a condition slightly stronger than (5.1). In [32] he also obtained sufficient and necessary results on circle-like behavior involving modules of ring domains. Later, Wittich [33] showed that (5.3) holds for quasiconformal maps satisfying (5.1). This result is sometimes referred to as Teichmüller-Wittich theorem. In 1954 Belinskii [3] proved that for a general quasiconformal mapping $f$ with real dilatation $D_{f},(5.1)$ implies (5.3) and for an appropriate choice of the argument

$$
\lim _{z \rightarrow 0} \arg \frac{f(z)}{z}=a
$$

as well, thus proving conformality at a point when (5.1) holds.

Reich and Walczak [29] studied conformality at a point as well. Among other interesting results they showed the following theorem.

Theorem 5.3. [29] Let $f$ be a quasiconformal mapping and $\theta=\arg z$. If

$$
\iint_{U}\left|D_{f, \theta}-1\right| \frac{d \theta d r}{r}<\infty \text { and } \iint_{U}\left|D_{f, \theta+\frac{\pi}{2}}-1\right| \frac{d \theta d r}{r}<\infty
$$

then (5.3) holds.

Theorem 5.3 extends Teichmüller-Wittich theorem. The authors [29] show that (5.5) is not sufficient to claim convergence of the argument (5.4), as $\arg f\left(r e^{i \theta}\right)$ may go to $\infty$ as $r \rightarrow 0$ at a rate as big as $o(\sqrt{\log (1 / r)})$, where the estimate is sharp. It seems that one could easily extend this result to the weaker case of $\mu$-homeomorphisms when (1.4) is finite for $\alpha=0, \pi / 2$. 
Regularity conditions for $K$-quasiconformal mappings were also studied by Lehto $[23,25]$.

Circle-like behavior and regularity properties were studied in 1988 by the author $[6,7]$. An equivalent version of a generalization of the Teichmüller-Wittich theorem, from these works, in the notations of this paper is stated below.

Theorem 5.4. [7] Let $f$ be a continuously differentiable $\mu$-homeomorphism. If

$$
\iint_{U} \frac{\left|\mu_{f}\right|^{2}}{1-\left|\mu_{f}\right|^{2}} \frac{d A_{z}}{|z|^{2}}<\infty
$$

then $f$ is circle-like, i.e. (1.7) holds. In addition, there exists a constant $A>0$ such that

$$
\left|f\left(r e^{i \theta}\right)\right| \sim A \exp \left[-\iint_{r<|z|<1} D_{f, \theta} \frac{d A_{z}}{|z|^{2}}\right] \quad \text { as } r \rightarrow 0,
$$

where $D_{f, \theta}$ is the radial dilatation at $z=r e^{i \theta}$.

As shown in [6, 7], if (5.1) holds then there exist a constant $A_{0}$ such that

$$
\exp \left[-\iint_{r<|z|<1} D_{f, \theta} \frac{d A_{z}}{|z|^{2}}\right] \sim A_{0} r \quad \text { as } r \rightarrow 0,
$$

and the Teichmüller-Wittich theorem follows in the case of continuously differentiable $\mu$-homeomorphisms. Consider the radial stretching $f\left(r e^{i \theta}\right)=\frac{r e^{i \theta}}{1+\log r}$ with $\mu_{f}\left(r e^{i \theta}\right)=\frac{-e^{2 i \theta}}{1+2 \log r}$. For this map the integral in (5.2) diverges, while (5.6) holds.

Sufficient conditions for asymptotic rotation on circles for $\mu$-homeomorphisms, were first obtained by the author and Jenkins in 1994 [9].

Theorem 5.5. [9]. Assume that $f$ is an a.e. regular $\mu$-homeomorphism in $U$, $f(0)=0$. Let $\theta=\arg z$. Assume that $D_{f, \theta+\alpha} \in L^{1}\left(A\left(r_{2}, r_{1}\right)\right)$ for $\alpha=0, \pi / 2$. If

$$
\iint_{U} \frac{\left|\mu_{f}\right|^{2}+\left|\Re e^{-2 i \theta} \mu_{f}\right|}{1-\left|\mu_{f}\right|^{2}} \frac{d A_{z}}{|z|^{2}}<\infty,
$$

then (5.3) holds and $f$ is asymptotically a rotation on circles at $z=0$.

A strip version of Theorem 5.1 that applies to a general class of $\mu$-homeomorphisms is proven in [9] as Lemma 6.1. From that result follows the extension of the Teichmüller-Wittich-Belinskii Theorem to the class of $\mu$-homeomorphisms, asserting conformality under the condition

$$
\iint_{U} \frac{|\mu|}{1-|\mu|} \frac{d A_{z}}{|z|^{2}}<\infty
$$

A recent breakthrough (2003) in relaxing the analytic conditions for conformality at a point, in the case of a $K$-quasiconformal mapping, was obtained in a paper of Gutlyanskii and Martio [18]. 
Theorem 5.6. [18] Let $f$ be a $K$-quasiconformal homeomorphism in $U$ such that $f(0)=0$. If

$$
\iint_{U} \frac{\left|\mu_{f}\right|^{2}}{|z|^{2}} d A_{z}<\infty
$$

and if the singular integral

$$
\iint_{U} \frac{\mu_{f}}{z^{2}} d A_{z}
$$

exists in the sense of principal value, then $f$ is conformal at $z=0$.

The proof of Theorem 5.6 follows a somewhat unusual path. First, the authors show that (5.9) implies weak conformality at the origin (see Section 1). If, in addition, (5.10) holds, then one has convergence of the argument (5.4). In the proof of Theorem 5.6 one uses deep results from the theory of $K$-quasiconformal mappings that do not necessarily extend to the class of $\mu$-homeomorphisms considered here. The following remark, based on [18, Theorem 1.7], shows to what extent Theorem 5.6 extends Theorem 5.1 within the class of $K$-quasiconformal mappings.

Remark 5.7. [18] For each $K$-quasiconformal mapping $f$ whose complex dilatation $\mu_{f}$ satisfies (5.9) and does not satisfy (5.2) one can construct a $K$-quasiconformal mapping $g$ such that $\left|\mu_{f}\right|=\left|\mu_{g}\right|$ a.e. in $U$ such that $g$ satisfies (5.9) and (5.10) (and thus is conformal at the origin).

Using any of the existence theorems for the degenerate complex Beltrami equation in the planar case, e.g. $[8,11,19,20,31]$, and others, one can modify the proof of Remark 5.7 so that it holds for the general class of $\mu$-homeomorphisms for which the existence theorems apply.

Note that Theorem 5.6 is the quasiconformal version of Theorem 4.5 (Theorem 1.3, respectively), since if $f$ is $K$-quasiconformal, (4.6) and (4.8) are equivalent to (5.9) and (5.10), respectively. The quasiconformal counterpart of Theorem 4.4, and thus Theorem 1.2, implying (5.3), was also first proven in [18]. An "uniform" version of this result is given in [17]. Properties like asymptotic rotation on circles, which is stronger than weak conformality, (1.9), and the quasiconformal counterpart of Theorem 1.4 and Theorem 4.6, respectively, seem to have not been directly addressed in [18].

Below we give a couple of examples of $\mu$-homeomorphisms, conformal at the origin, such that $\limsup _{r \rightarrow 0}\left|\mu_{f}\left(r e^{i \theta}\right)\right| \rightarrow 1$ and thus $\left\|\mu_{f}\right\|_{\infty}=1$ in any neighborhood of the origin.

Example 5.8. Let $G=\bigcup_{n=1}^{\infty} G_{n}$, where $G_{n}=\frac{1}{n} \leq|z|<\frac{1}{n} e^{1 / n^{3}}$. We consider the radial stretching $f(z)=\rho(r) e^{i \theta}$, where $\rho(r)=r$ if $z \in U \backslash G$, and

$$
\rho(r)=\frac{e^{\frac{1}{n^{3}}}-1}{n}\left\{\left[\frac{n}{n+2}\left(\frac{n r-1}{\left(e^{1 / n^{3}}-1\right)}+\frac{1}{n}\right)^{2}+\frac{n^{2}+2 n-1}{n^{2}+2 n}\right]-1\right\}+\frac{1}{n}
$$

if $z \in G_{n}$. 
Observe that $\rho(r)$ simplifies to $\frac{(n r-1)^{2}+\left(e^{1 / n^{3}}-1\right)(2 r+1)}{\left(e^{1 / n^{3}}-1\right)(n+2)}, \mu_{f}\left(r e^{i \theta}\right)=0$ in $U \backslash G$ and $\mu_{f}\left(r e^{i \theta}\right)=\frac{r \rho^{\prime}-\rho}{r \rho^{\prime}+\rho} e^{2 i \theta}$ in $G$. In each $G_{n},\left|\mu_{f}\right|$ attains its maximum value of $\left|\mu_{f}\right|=\frac{n}{4+n}$ at $|z|=1 / n$. Thus $\limsup _{r \rightarrow 0}\left|\mu_{f}\left(r e^{i \theta}\right)\right| \rightarrow 1$ and therefore $f$ is not $K$-quasiconformal. Since (5.8) holds,

$$
\iint_{U} \frac{\left|\mu_{f}\right|}{1-\left|\mu_{f}\right|^{2}} \frac{d A_{z}}{|z|^{2}} \leq 2 \pi \sum_{n=1}^{\infty} \frac{1}{1-\left|\mu_{f}\right|} \frac{1}{n^{3}} \leq 2 \pi \sum_{n=1}^{\infty} \frac{1+n / 4}{n^{3}}<\infty,
$$

it follows that $f$ is conformal at the origin. The conformality of this radial stretching follows directly from the construction as well.

Example 5.9. Define a decreasing sequence $\left\{r_{n}\right\}_{n=1}^{\infty}$ as follows: $r_{1}=1, r_{2}=$ $\frac{1}{e}, r_{2 n+1}=\left(r_{2 n}\right)^{n} \sqrt{e}, r_{2 n+2}=r_{2 n+1} e^{-1 / n^{3}}$ for $n=1,2, \ldots$. Define a Beltrami coefficient $\mu$ in $U$ such that $\mu=0$ in $\left(\frac{1}{e}, 1\right), \mu\left(r e^{i \theta}\right)=\frac{(-1)^{n+1} e^{2 i \theta}}{\ln r}$ in $\left(r_{2 n+1}, r_{2 n}\right], \mu\left(r e^{i \theta}\right)=$ $\frac{n-1}{n} e^{2 i \theta}$ in $\left(r_{2 n+2}, r_{2 n+1}\right]$, and $\mu(0)=0$. Since $\mu$ is subexponentially integrable [20], there exists a $\mu$-homeomorphism $f, f(0)=0,[14,8,20]$, such that $\mu_{f}=\mu$ a.e.

Clearly, in the above example, $\limsup _{r \rightarrow 0}\left|\mu_{f}\left(r e^{i \theta}\right)\right| \rightarrow 1$. After some computations one can verify that $\mu_{f}$ satisfies the conditions of Theorems 4.4-4.5, Theorems 1.2-1.3, respectively, and therefore $f$ is conformal at 0 . Also $f$ does not satisfy any of the conditions (5.1), (5.5), (5.7) and since, in addition, $f$ is not $K$-quasiconformal, one can not apply Theorem 5.1, Theorem 5.3, Theorem 5.5 and Theorem 5.6. Observe that (5.8) does not hold either, while (5.6) holds. Thus we have extended most of the results on conformality and related notions discussed in this section within the class of $K$-quasiconformal maps and to a general class of $\mu$-homeomorphisms.

\section{References}

[1] Ahlfors, L. V.: Lectures on quasiconformal mappings. - Univ. Lecture Ser. 38, 2nd ed. (with additional chapters by C. J. Earle and I. Kra, M. Shishikura, J. Hubbard), Amer. Math. Soc., 2006.

[2] Ahlfors, L. V.: Conformal invariants: Topics in geometric function theory. - McGraw-Hill, 1973.

[3] Belinskit, P. P.: Behavior of a quasiconformal mapping at an isolated singular point. - Lvov Gos. Univ. Uchen. Zap. Ser. Meh.-Mat. 29, 1954, 58-70 (in Russian).

[4] Belinskit, P.: General properties of quasiconformal mappings. - Nauka, Novosibirsk, 1974 (in Russian).

[5] Bojarski, B. V.: Generalized solutions of a system of differential equations of the first order and elliptic type with discontinuous coefficients. - Report University of Jyväskylä Dept. of Math. and Stat. 118, transl. from the 1957 Russian original, with a forward by E. Saksman, 2009.

[6] Bracalova, M.: A generalization of the Teichmüller theorem. - In: Complex Analysis (Joensuu 1987), Lecture Notes in Math. 1351, Springer, 1988, 69-77.

[7] Brakalova, M.: On the asymptotic behavior of some conformal and quasiconformal mappings. - Thesis, Sofia University, Sofia, 1988, 1-115. 
[8] Brakalova, M. A., and J. A. Jenkins: On solutions of the Beltrami equation. - J. Anal. Math. 76, 1998, 67-92.

[9] Brakalova, M., and J. A. Jenkins: On the local behavior of certain homeomorphisms. Kodai Math. J. 17, 1994, 201-213.

[10] Brakalova, M., and J. A. Jenkins: On a paper of Carleson. - Ann. Acad. Sci. Fenn. Math. 27, 2002, 485-490.

[11] Brakalova, M. A., and J. A. Jenkins: On solutions of the Beltrami equation II. - Publ. Inst. Math. (Beograd) (N.S.) 89, 2004, 3-8.

[12] Brakalova, M.: Sufficient and necessary conditions for conformality. Part I. Geometric viewpoint. - Complex Var. Elliptic Equ. (to appear).

[13] Andreian Cazacu, C.: On the length-area dilatation. - Complex Var. Theory Appl. 50, 2005, $1-21$.

[14] David, G.: Solutions de léquation de Beltrami avec $\|\mu\|_{\infty}=1$. - Ann. Acad. Sci. Fenn. Ser. A I Math. 13, 1988, 25-70.

[15] Drasin, D.: On the Teichmüller-Wittich-Belinskii theorem. - Results Math. 10, 1986, 54-65.

[16] Goldberg, A., and I. Ostrovskir: Value distribution of meromorphic functions. - Transl. Math. Monogr. 236 (translated from the 1970 original by M. Ostrovskii, with an appendix by A. Eremenko and J. Langley), AMS, Providence, RI, 2008.

[17] Gutlyanskit, V., and O. Martio: On regularity of quasiconformal curves. - Comput. Methods Funct. Theory 2, 2002, 367-384.

[18] Gutlyanskit, V., and O. Martio: Conformality of a quasiconformal mapping at a point. J. Anal. Math. 91, 2003, 179-191.

[19] Gutlyanskit, V., O. Martio, T. Sugawa, and M. Vuorinen: On the degenerate Beltrami equation. - Trans. Amer. Math. Soc. 357:3, 2005, 875-900.

[20] Iwaniec, T., and G. Martin: Geometric function theory and non-linear analysis. - Clarendon Press, Oxford, 2001.

[21] Jenkins, J.A.: Univalent functions and conformal mapping. - Springer Verlag, BerlinGöttingen-Heidelberg, 1958.

[22] Kovalev, L.: Quasiregular mappings of maximal local modulus of continuity. - Ann. Acad. Sci. Fenn. Math. 29, 2004, 211-222.

[23] Lehto, O.: On the differentiability of quasiconformal mappings with prescribed complex dilatation. - Ann. Acad. Sci. Fenn. Ser. A I Math. 275, 1960, 1-28.

[24] Lehto, O.: Homeomorphisms with a prescribed dilatation. - Lecture Notes in Math. 118, $1968,58-73$.

[25] Lehto, O., and K. I. Virtanen: Quasiconformal mappings in the plane. - Springer Verlag, 1973.

[26] Volkovyskit, L.: Investigation of the type problem for a simply connected Riemann surface. - Trudy Mat. Inst. Steklov 34, 1950 (in Russian).

[27] Martio, O., V. Ryazanov, U. Srebro, and E. Yakubov: Moduli in modern mapping theory. - Springer Monogr. Math., Springer, New York, 2009.

[28] Monroe, M. E.: Measure and integration. - Addison-Wesley, 1953.

[29] Reich, E., and H. WalczaK: On the behavior of quasiconformal mappings at a point. Trans. Amer. Math. Soc. 117, 1965, 338-351.

[30] Rodin, B.: The method of extremal length. - Bull. Amer. Math. Soc. 80, 1974, 587-606.

[31] Ryazanov, V., U. Srebro, and E. Yakubov: BMO-quasiconformal mappings. - J. Anal. Math. 83, 2001, 1-20. 
[32] Teichmüller, O.: Untersuchungen über konforme und qusiconforme Abbildung. - Deutsche Math. 3, 1938, 621-678.

[33] Wiтtich, H.: Zum Beweis eines Satzes über quasikonforme Abbildungen. - Math. Z. 51, 1948, 275-288.

Received 23 February 2009 